\title{
Rubinstein-Taybi Syndrome Type 2
}

National Cancer Institute

\section{Source}

National Cancer Institute. Rubinstein-Taybi Syndrome Type 2. NCI Thesaurus. Code C153291.

Rubinstein-T aybi syndrome caused by a mutation in the EP300 gene on chromosome $22 q 13$, which presents with a mild phenotype associated with less severe facial dysmorphism and better cog nitive function. 